\title{
THE NEUMANN PROBLEM FOR NONLOCAL NONLINEAR DIFFUSION EQUATIONS
}

\author{
FUENSANTA ANDREU, JOSÉ M. MAZÓN, JULIO D. ROSSI AND JULIÁN TOLEDO
}

ABstract. We study nonlocal diffusion models of the form

$$
(\gamma(u))_{t}(t, x)=\int_{\Omega} J(x-y)(u(t, y)-u(t, x)) d y .
$$

Here $\Omega$ is a bounded smooth domain and $\gamma$ is a maximal monotone graph in $\mathbb{R}^{2}$. This is a nonlocal diffusion problem analogous with the usual Laplacian with Neumann boundary conditions. We prove existence and uniqueness of solutions with initial conditions in $L^{1}(\Omega)$. Moreover, when $\gamma$ is a continuous function we find the asymptotic behaviour of the solutions, they converge as $t \rightarrow \infty$ to the mean value of the initial condition.

\section{INTRODUCTION}

First, let us introduce what kind of nonlocal diffusion problems we consider. Nonlocal evolution equations of the form

$$
u_{t}(t, x)=J * u-u(t, x)=\int_{\mathbb{R}^{N}} J(x-y) u(t, y) d y-u(t, x),
$$

and variations of it, have been recently widely used to model diffusion processes, see $[2],[4],[9],[11],[12],[16],[17],[18],[19]$ and [20]. As stated in [16], if $u(t, x)$ is thought of as the density of a single population at the point $x$ at time $t$, and $J(x-y)$ is thought of as the probability distribution of jumping from location $y$ to location $x$, then the convolution $(J * u)(t, x)=\int_{\mathbb{R}^{N}} J(y-x) u(t, y) d y$ is the rate at which individuals are arriving to position $x$ from all other places and $-u(t, x)=-\int_{\mathbb{R}^{N}} J(y-x) u(t, x) d y$ is the rate at which they are leaving location $x$ to travel to all other sites. This consideration, in the absence of external or internal sources, leads immediately to the fact that the density $u$ satisfies equation (1.1). Moreover, a nonlinearity of the form $\int J(x-y)(F(u(y))-F(u(x))) d y$ may also appear in population models, see [9] and references therein.

Equation (1.1) is called nonlocal diffusion equation since the diffusion of the density $u$ at a point $x$ and time $t$ does not only depend on $u(t, x)$, but on all the values of $u$ in a neighborhood of $x$ through the convolution term $J * u$. This equation shares many properties with the classical heat equation, $u_{t}=c u_{x x}$, such as: bounded stationary solutions are constant, a maximum principle holds for both of them and, even if $J$ is compactly supported, perturbations propagate with infinite speed, [16]. However, there is no regularizing effect in general.

\footnotetext{
Key words and phrases. Nonlocal diffusion, Neumann boundary conditions, asymptotic behaviour.

2000 Mathematics Subject Classification. 35B40, 35A07, 45G10.
} 
In this paper we turn our attention to nonlinear evolutions with Neumann boundary conditions. We study

$$
P_{\gamma}^{J}\left(z_{0}\right) \begin{cases}z_{t}(t, x)=\int_{\Omega} J(x-y)(u(t, y)-u(t, x)) d y, & x \in \Omega, t>0, \\ z(t, x) \in \gamma(u(t, x)), & x \in \Omega, t>0, \\ z(0, x)=z_{0}(x), & x \in \Omega .\end{cases}
$$

Here $\Omega$ is a bounded domain, $z_{0} \in L^{1}(\Omega), \gamma$ is a maximal monotone graph in $\mathbb{R}^{2}$ such that $0 \in \gamma(0)$, and $J: \mathbb{R}^{N} \rightarrow \mathbb{R}$ is a nonnegative continuous radial function with $\int_{\mathbb{R}^{N}} J(r) d r=1$ and $0 \in \operatorname{int}[\operatorname{supp}(J)]$.

In this model the right hand side takes into account the diffusion inside $\Omega$. In fact, as we have explained, the integral $\int J(x-y)(u(t, y)-u(t, x)) d y$ takes into account the individuals arriving or leaving position $x$ from or to other places. Since we are integrating in $\Omega$, we are imposing that diffusion takes place only in $\Omega$. There is no flux of individuals across the boundary. This is the analogous of what is called homogeneous Neumann boundary conditions in the literature.

Solutions to $P_{\gamma}^{J}\left(z_{0}\right)$ will be understood in the following sense.

Definition 1.1. A solution of $P_{\gamma}^{J}\left(z_{0}\right)$ in $[0, T]$ is a function $z \in W^{1,1}(] 0, T\left[; L^{1}(\Omega)\right)$ which satisfies $z(0, x)=z_{0}(x)$, a.e. $x \in \Omega$, and for which there exists $u \in L^{2}\left(0, T ; L^{2}(\Omega)\right)$, $z \in \gamma(u)$ a.e. in $\left.Q_{T}=\Omega \times\right] 0, T[$, such that

$$
\left.z_{t}(t, x)=\int_{\Omega} J(x-y)(u(t, y)-u(t, x)) d y \quad \text { a.e in } \quad\right] 0, T[\times \Omega .
$$

Our main results can be summarized as follows.

"Under some natural assumptions about the initial condition $z_{0}$, there exists a unique global solution to $P_{\gamma}^{J}\left(z_{0}\right)$. Moreover, a contraction principle holds, given two solutions $z_{i}$ of $P_{\gamma}^{J}\left(z_{i 0}\right), i=1,2$, then

$$
\int_{\Omega}\left(z_{1}(t)-z_{2}(t)\right)^{+} \leq \int_{\Omega}\left(z_{10}-z_{20}\right)^{+} .
$$

Respect to the asymptotic behaviour of the solution we prove that if $\gamma$ is a continuous function, then

$$
\lim _{t \rightarrow \infty} z(t)=\frac{1}{|\Omega|} \int_{\Omega} z_{0}
$$

strongly in $L^{1}(\Omega)$ ".

Concerning previous references for this model, we cite [10], [12] and [13] where only the linear case, $\gamma(s)=s$, was considered. In [10] and [12] the asymptotic behaviour for the linear model is studied and in [13] it is proved that solutions to the linear problem converge to the solution of the classical heat equation with Neumann boundary conditions when the convolution kernel $J$ is rescaled in a suitable way.

We can consider different maximal monotone graphs $\gamma$. For example, if $\gamma(r)=r^{m}$, problem $P_{\gamma}^{J}\left(z_{0}\right)$ corresponds to the nonlocal version of the porous medium (or fast diffusion) problems. Note also that $\gamma$ may be multivalued, so we are considering the 
nonlocal version of various phenomena with phase changes like the multiphase Stefan problem, for which

$$
\gamma(r)= \begin{cases}r-1 & \text { if } \quad r<0 \\ {[-1,0]} & \text { if } r=0 \\ r & \text { if } r>0\end{cases}
$$

Even $\gamma$ can have a domain different from $\mathbb{R}$, which corresponds to obstacle problems.

We have to point out that the right hand side of the equation in $P_{\gamma}^{J}\left(z_{0}\right)$ has no regularizing effect. Hence the analysis becomes more involved (due to the lack of compactness) that the one that can be done for the usual Laplacian with Neumann boundary conditions.

Organization of the paper. The rest of the paper is organized as follows: in Section 2 we collect some preliminaries and fix the notation. In Section 3, by means of Nonlinear Semigroup Theory, we construct a mild solution and we prove uniqueness of solutions, in fact we prove a contraction principle. In Section 4 we prove that the mild solution is indeed a solution in the sense of Definition 1.1. Finally, Section 5 deal with the asymptotic behaviour of the solutions.

\section{Notations AND PRELIMINARIES}

In this section we collect some preliminaries and notations that will be used in the sequel. For a maximal monotone graph $\eta$ in $\mathbb{R} \times \mathbb{R}$ and $r \in \mathbb{N}$ we denote by $\eta_{r}$ the Yosida approximation of $\eta$, given by $\eta_{r}=r\left(I-\left(I+\frac{1}{r} \eta\right)^{-1}\right)$. The function $\eta_{r}$ is maximal monotone and Lipschitz. We recall the definition of the main section $\eta^{0}$ of $\eta$

$$
\eta^{0}(s):= \begin{cases}\text { the element of minimal absolute value of } \eta(s) \text { if } \eta(s) \neq \emptyset \\ +\infty \quad \text { if }[s,+\infty) \cap D(\eta)=\emptyset \\ -\infty \quad \text { if }(-\infty, s] \cap D(\eta)=\emptyset\end{cases}
$$

where $D(\eta)$ denotes the domain of $\eta$. The following properties hold: if $s \in D(\eta)$, $\left|\eta_{r}(s)\right| \leq\left|\eta^{0}(s)\right|$ and $\eta_{r}(s) \rightarrow \eta^{0}(s)$ as $r \rightarrow+\infty$, and if $s \notin D(\eta),\left|\eta_{r}(s)\right| \rightarrow+\infty$ as $r \rightarrow+\infty$.

We will use the following notations, $\eta_{-}:=\inf \operatorname{Ran}(\eta)$ and $\eta_{+}:=\sup \operatorname{Ran}(\eta)$, where $\operatorname{Ran}(\eta)$ denotes the range of $\eta$. If $0 \in D(\eta), j_{\eta}(r)=\int_{0}^{r} \eta^{0}(s) d s$ defines a convex l.s.c. function such that $\eta=\partial j_{\eta}$. If $j_{\eta}^{*}$ is the Legendre transform of $j_{\eta}$ then $\eta^{-1}=\partial j_{\eta}^{*}$.

Also we will denote by $J_{0}$ and $P_{0}$ the following sets of functions,

$$
\begin{gathered}
J_{0}=\{j: \mathbb{R} \rightarrow[0,+\infty], \text { convex and lower semi-continuos with } j(0)=0\}, \\
P_{0}=\left\{p \in C^{\infty}(\mathbb{R}): 0 \leq p^{\prime} \leq 1, \operatorname{supp}\left(p^{\prime}\right) \text { is compact, and } 0 \notin \operatorname{supp}(p)\right\} .
\end{gathered}
$$

In [6] the following relation for $u, v \in L^{1}(\Omega)$ is defined,

$$
u \ll v \text { if and only if } \int_{\Omega} j(u) d x \leq \int_{\Omega} j(v) d x,
$$

and the following facts are proved.

Proposition 2.1. Let $\Omega$ be a bounded domain in $\mathbb{R}^{N}$.

(i) For any $u, v \in L^{1}(\Omega)$, if $\int_{\Omega} u p(u) \leq \int_{\Omega} v p(u)$ for all $p \in P_{0}$, then $u \ll v$.

(ii) If $u, v \in L^{1}(\Omega)$ and $u \ll v$, then $\|u\|_{q} \leq\|v\|_{q}$ for any $q \in[1,+\infty]$. 
(iii) If $v \in L^{1}(\Omega)$, then $\left\{u \in L^{1}(\Omega): u \ll v\right\}$ is a weakly compact subset of $L^{1}(\Omega)$.

The following Poincaré's type inequality is given in [10].

Proposition 2.2. Given $J$ and $\Omega$ the quantity

$$
\beta_{1}:=\beta_{1}(J, \Omega)=\inf _{u \in L^{2}(\Omega), \int_{\Omega} u=0} \frac{\frac{1}{2} \int_{\Omega} \int_{\Omega} J(x-y)(u(y)-u(x))^{2} d y d x}{\int_{\Omega}(u(x))^{2} d x}
$$

is strictly positive. Consequently

$$
\beta_{1} \int_{\Omega}\left|u-\frac{1}{|\Omega|} \int_{\Omega} u\right|^{2} \leq \frac{1}{2} \int_{\Omega} \int_{\Omega} J(x-y)(u(y)-u(x))^{2} d y d x, \quad \forall u \in L^{2}(\Omega)
$$

In order to obtain a generalized Poincaré's type inequality we need the following result.

Proposition 2.3. Let $\Omega \subset \mathbb{R}^{N}$ be a bounded open set and $k>0$. There exists a constant $C>0$ such that for any $K \subset \Omega$ with $|K|>k$, it holds

$$
\|u\|_{L^{2}(\Omega)} \leq C\left(\left\|u-\frac{1}{|\Omega|} \int_{\Omega} u\right\|_{L^{2}(\Omega)}+\left|\int_{K} u\right|\right), \quad \forall u \in L^{2}(\Omega) .
$$

Proof. Assume the conclusion does not hold. Then, for every $n \in \mathbb{N}$ there exists $K_{n} \subset \Omega$ with $\left|K_{n}\right|>k$, and $u_{n} \in L^{2}(\Omega)$ satisfying

$$
\left\|u_{n}\right\|_{L^{2}(\Omega)} \geq n\left(\left\|u_{n}-\frac{1}{|\Omega|} \int_{\Omega} u_{n}\right\|_{L^{2}(\Omega)}+\left|\int_{K_{n}} u_{n}\right|\right), \quad \forall n \in \mathbb{N} .
$$

We normalize $u_{n}$ by $\left\|u_{n}\right\|_{L^{2}(\Omega)}=1$ for all $n \in \mathbb{N}$, and consequently we can assume that

$$
u_{n} \rightarrow u \quad \text { weakly in } L^{2}(\Omega) .
$$

Moreover, by (2.4), we have

$$
\left\|u_{n}-\frac{1}{|\Omega|} \int_{\Omega} u_{n}\right\|_{L^{2}(\Omega)} \leq \frac{1}{n}, \quad \text { and } \quad\left|\int_{K_{n}} u_{n}\right| \leq \frac{1}{n}, \quad \forall n \in \mathbb{N} .
$$

Hence

$$
u_{n}-\frac{1}{|\Omega|} \int_{\Omega} u_{n} \rightarrow 0 \quad \text { in } L^{2}(\Omega)
$$

and by (2.5) we get $u(x)=\frac{1}{|\Omega|} \int_{\Omega} u=\alpha$ for almost all $x \in \Omega$, and $u_{n} \rightarrow \alpha$ strongly in $L^{2}(\Omega)$. Since $\left\|u_{n}\right\|_{L^{2}(\Omega)}=1$ for each $n \in \mathbb{N}, \alpha \neq 0$. On the other hand, (2.6) implies

$$
\lim _{n \rightarrow \infty} \int_{K_{n}} u_{n}=0
$$

Since $\chi_{K_{n}}$ is bounded in $L^{2}(\Omega)$, we can extract a subsequence (still denoted by $\chi_{K_{n}}$ ) such that

$$
\chi_{K_{n}} \rightarrow \phi \quad \text { weakly in } L^{2}(\Omega)
$$


Moreover, $\phi$ is nonnegative and verifies

$$
k \leq \lim _{n \rightarrow \infty}\left|K_{n}\right|=\lim _{n \rightarrow \infty} \int_{\Omega} \chi_{K_{n}}=\int_{\Omega} \phi
$$

Now, since $u_{n} \rightarrow \alpha$ strongly in $L^{2}(\Omega)$ and $\chi_{K_{n}} \rightarrow \phi$ weakly in $L^{2}(\Omega)$ we have

$$
0=\lim _{n \rightarrow \infty} \int_{K_{n}} u_{n}=\lim _{n \rightarrow \infty} \int_{\Omega} \chi_{K_{n}} u_{n}=\alpha \int_{\Omega} \phi \neq 0
$$

a contradiction.

To simplify the notation we define the linear self-adjoint operator $A: L^{2}(\Omega) \rightarrow$ $L^{2}(\Omega)$ by

$$
A u(x)=\int_{\Omega} J(x-y)(u(y)-u(x)) d y, \quad x \in \Omega .
$$

As a consequence of the above results we have the next proposition, which plays the role of the classical generalized Poincaré's inequality for Sobolev spaces.

Proposition 2.4. Let $\Omega \subset \mathbb{R}^{N}$ be a bounded open set and $k>0$. There exists a constant $C=C(J, \Omega, k)$ such that, for any $K \subset \Omega$ with $|K|>k$,

$$
\|u\|_{L^{2}(\Omega)} \leq C\left(\left(-\int_{\Omega} A u u\right)^{1 / 2}+\|u\|_{L^{2}(K)}\right) \quad \forall u \in L^{2}(\Omega) .
$$

Using the above result and working as in the proof of Lemma 4.2 in [1], we obtain the following lemma, of which we give the proof for the sake of completeness.

Lemma 2.5. Let $\gamma$ be a maximal monotone graph in $\mathbb{R}^{2}$ such that $0 \in \gamma(0)$. Let $\left\{u_{n}\right\}_{n \in \mathbb{N}} \subset L^{2}(\Omega)$ and $\left\{z_{n}\right\}_{n \in \mathbb{N}} \subset L^{1}(\Omega)$ such that, for every $n \in \mathbb{N}, z_{n} \in \gamma\left(u_{n}\right)$ a.e. in $\Omega$.

Let us suppose that

(i) if $\gamma_{+}=+\infty$, there exists $M>0$ such that

$$
\int_{\Omega} z_{n}^{+}<M, \quad \forall n \in \mathbb{N},
$$

(ii) if $\gamma_{+}<+\infty$, there exists $M \in \mathbb{R}$ and $h>0$ such that

$$
\int_{\Omega} z_{n}<M<\gamma_{+}|\Omega|, \quad \forall n \in \mathbb{N}
$$

and

$$
\int_{\left\{x \in \Omega: z_{n}(x)<-h\right\}}\left|z_{n}\right|<\frac{\gamma_{+}|\Omega|-M}{4}, \quad \forall n \in \mathbb{N} .
$$

Then, there exists a constant $C=C(M, \Omega)$ in case (i), $C=C(M, \Omega, \gamma, h)$ in case (ii), such that

$$
\left\|u_{n}^{+}\right\|_{L^{2}(\Omega)} \leq C\left(\left(-\int_{\Omega} A u_{n}^{+} u_{n}^{+}\right)^{1 / 2}+1\right), \quad \forall n \in \mathbb{N} .
$$

Let us suppose that 
(iii) if $\gamma_{-}=-\infty$, there exists $M>0$ such that

$$
\int_{\Omega} z_{n}^{-}<M, \quad \forall n \in \mathbb{N}
$$

(iv) if $\gamma_{-}>-\infty$, there exists $M \in \mathbb{R}$ and $h>0$ such that

$$
\int_{\Omega} z_{n}>M>\gamma_{-}|\Omega|, \quad \forall n \in \mathbb{N}
$$

and

$$
\int_{\left\{x \in \Omega: z_{n}(x)>h\right\}} z_{n}<\frac{M-\gamma_{-}|\Omega|}{4}, \quad \forall n \in \mathbb{N} .
$$

Then, there exists a constant $\tilde{C}=\tilde{C}(M, \Omega)$ in case (iii), $\tilde{C}=\tilde{C}(M, \Omega, \gamma, h)$ in case (iv), such that

$$
\left\|u_{n}^{-}\right\|_{L^{2}(\Omega)} \leq \tilde{C}\left(\left(-\int_{\Omega} A u_{n}^{-} u_{n}^{-}\right)^{1 / 2}+1\right), \quad \forall n \in \mathbb{N} .
$$

Proof. Let us only prove (2.8), since the proof of (2.9) is similar. First, consider the case $\gamma_{+}=+\infty$. Then, by assumption, there exists $M>0$ such that

$$
\int_{\Omega} z_{n}^{+}<M, \quad \forall n \in \mathbb{N} .
$$

For $n \in \mathbb{N}$ let $K_{n}=\left\{x \in \Omega: z_{n}^{+}(x)<\frac{2 M}{|\Omega|}\right\}$. Then

$$
0 \leq \int_{K_{n}} z_{n}^{+}=\int_{\Omega} z_{n}^{+}-\int_{\Omega \backslash K_{n}} z_{n}^{+} \leq M-\left(|\Omega|-\left|K_{n}\right|\right) \frac{2 M}{|\Omega|}=\left|K_{n}\right| \frac{2 M}{|\Omega|}-M .
$$

Therefore,

and

$$
\left|K_{n}\right| \geq \frac{|\Omega|}{2}
$$

$$
\left\|u_{n}^{+}\right\|_{L^{2}\left(K_{n}\right)} \leq\left|K_{n}\right|^{1 / 2} \sup \gamma^{-1}\left(\frac{2 M}{|\Omega|}\right) .
$$

Then, by Proposition 2.4, for all $n \in \mathbb{N}$,

$$
\left\|u_{n}^{+}\right\|_{L^{2}(\Omega)} \leq \tilde{C}(J, \Omega)\left(\left(-\int_{\Omega} A u_{n}^{+} u_{n}^{+}\right)^{1 / 2}+|\Omega|^{1 / 2} \sup \gamma^{-1}\left(\frac{2 M}{|\Omega|}\right)\right) .
$$

Now, let us consider the case $\gamma_{+}<+\infty$. Let

$$
\delta=\gamma_{+}|\Omega|-M
$$

By assumption, for every $n \in \mathbb{N}$, we have,

$$
\int_{\Omega} z_{n}<\gamma_{+}|\Omega|-\delta
$$

For $n \in \mathbb{N}$, let $K_{n}=\left\{x \in \Omega: z_{n}(x)<\gamma_{+}-\frac{\delta}{2|\Omega|}\right\}$. Then, by (2.10),

$$
\int_{K_{n}} z_{n}=\int_{\Omega} z_{n}-\int_{\Omega \backslash K_{n}} z_{n}<-\frac{\delta}{2}+\left|K_{n}\right|\left(\gamma_{+}-\frac{\delta}{2|\Omega|}\right) .
$$


Moreover,

$$
\int_{K_{n}} z_{n}=-\int_{K_{n} \cap\left\{x \in \Omega: z_{n}<-h\right\}}\left|z_{n}\right|+\int_{K_{n} \cap\left\{x \in \Omega: z_{n} \geq-h\right\}} z_{n} \geq-\frac{\delta}{4}-h\left|K_{n}\right| .
$$

Therefore,

$$
\left|K_{n}\right|\left(h-\frac{\delta}{2|\Omega|}+\gamma_{+}\right) \geq \frac{\delta}{4}
$$

Hence $\left|K_{n}\right|>0, h-\frac{\delta}{2|\Omega|}+\gamma_{+}>0$ and

$$
\left|K_{n}\right| \geq \frac{\delta}{4\left(h-\frac{\delta}{2|\Omega|}+\gamma_{+}\right)} .
$$

Consequently,

$$
\left\|u_{n}^{+}\right\|_{L^{2}\left(K_{n}\right)} \leq\left|K_{n}\right|^{1 / 2} \sup \gamma^{-1}\left(\gamma_{+}-\frac{\delta}{2|\Omega|}\right) .
$$

Then, by Proposition 2.4,

$$
\left\|u_{n}^{+}\right\|_{L^{2}(\Omega)} \leq \tilde{C}(J, \Omega, \gamma, h)\left(\left(-\int_{\Omega} A u_{n}^{+} u_{n}^{+}\right)^{1 / 2}+|\Omega|^{1 / 2} \sup \gamma^{-1}\left(\gamma_{+}-\frac{\delta}{2|\Omega|}\right)\right) .
$$

This ends the proof of (2.8).

Finally, we have the following monotonicity result. Its proof is straightforward.

Lemma 2.6. Let $T: \mathbb{R} \rightarrow \mathbb{R}$ a nondecreasing function. For every $u \in L^{2}(\Omega)$ such that $T(u) \in L^{2}(\Omega)$, it holds

$$
\begin{aligned}
-\int_{\Omega} A u(x) T(u(x)) d x & =-\int_{\Omega} \int_{\Omega} J(x-y)(u(y)-u(x)) d y T(u(x)) d x \\
& =\frac{1}{2} \int_{\Omega} \int_{\Omega} B(x, y)(u(y)-u(x))^{2} d y d x,
\end{aligned}
$$

where $B(x, y)$ is the non negative symmetric function given by

$$
B(x, y)= \begin{cases}J(x-y) \frac{T(u(y))-T(u(x))}{u(y)-u(x)} & \text { if } u(y) \neq u(x), \\ 0 & \text { if } u(y)=u(x) .\end{cases}
$$

In particular we have

$$
-\int_{\Omega} A u(x) u(x) d x=\frac{1}{2} \int_{\Omega} \int_{\Omega} J(x-y)(u(y)-u(x))^{2} d y d x .
$$

\section{Mild SOlutions AND CONTRACTION PRINCIPLE.}

In this section we obtain a mild solution to our problem studying the associated integral operator.

Given a maximal monotone graph $\gamma$ in $\mathbb{R}^{2}$ such that $0 \in \gamma(0), \gamma_{-}<\gamma_{+}$, we consider the problem,

$$
\left(S_{\phi}^{\gamma}\right) \quad \gamma(u)-A u \ni \phi \quad \text { in } \Omega
$$


Definition 3.1. Let $\phi \in L^{1}(\Omega)$. A pair of functions $(u, z) \in L^{2}(\Omega) \times L^{1}(\Omega)$ is a solution of problem $\left(S_{\phi}^{\gamma}\right)$ if $z(x) \in \gamma(u(x))$ a.e. $x \in \Omega$ and $z(x)-A u(x)=\phi(x)$ a.e. $x \in \Omega$, that is,

$$
z(x)-\int_{\Omega} J(x-y)(u(y)-u(x)) d y=\phi(x) \quad \text { a.e. } x \in \Omega .
$$

With respect to uniqueness of problem $\left(S_{\phi}^{\gamma}\right)$, we have the following maximum principle.

\section{Theorem 3.2.}

(i) Let $\phi_{1} \in L^{1}(\Omega)$ and $\left(u_{1}, z_{1}\right)$ a subsolution of $\left(S_{\phi_{1}}^{\gamma}\right)$, that is, $z_{1}(x) \in \gamma\left(u_{1}(x)\right)$ a.e. $x \in \Omega$ and $z_{1}(x)-A u_{1}(x) \leq \phi_{1}(x)$ a.e. $x \in \Omega$, and let $\phi_{2} \in L^{1}(\Omega)$ and $\left(u_{2}, z_{2}\right)$ a supersolution of $\left(S_{\phi_{2}}^{\gamma}\right)$, that is, $z_{2}(x) \in \gamma\left(u_{2}(x)\right)$ a.e. $x \in \Omega$ and $z_{2}(x)-A u_{2}(x) \geq \phi_{2}(x)$ a.e. $x \in \Omega$. Then

$$
\int_{\Omega}\left(z_{1}-z_{2}\right)^{+} \leq \int_{\Omega}\left(\phi_{1}-\phi_{2}\right)^{+} .
$$

Moreover, if $\phi_{1} \leq \phi_{2}, \phi_{1} \neq \phi_{2}$, then $u_{1}(x) \leq u_{2}(x)$ a.e. $x \in \Omega$.

(ii) Let $\phi \in L^{1}(\Omega)$, and $\left(u_{1}, z_{1}\right)$, $\left(u_{2}, z_{2}\right)$ two solutions of $\left(S_{\phi}^{\gamma}\right)$. Then, $z_{1}=z_{2}$ a.e. and there exists a constant $c$ such that $u_{1}=u_{2}+c$, a.e.

Proof. To prove $(i)$, let $\left(u_{1}, z_{1}\right)$ a subsolution of $\left(S_{\phi_{1}}^{\gamma}\right)$ and $\left(u_{2}, z_{2}\right)$ a supersolution of $\left(S_{\phi_{2}}^{\gamma}\right)$. Then

$$
-\left(A u_{1}(x)-A u_{2}(x)\right)+z_{1}(x)-z_{2}(x) \leq \phi_{1}(x)-\phi_{2}(x) .
$$

Multiplying the above inequality by $\frac{1}{k} T_{k}^{+}\left(u_{1}-u_{2}+k \operatorname{sign}_{0}^{+}\left(z_{1}-z_{2}\right)\right)$ and integrating we get,

$$
\begin{aligned}
& \int_{\Omega}\left(z_{1}-z_{2}\right) \frac{1}{k} T_{k}^{+}\left(u_{1}-u_{2}+k \operatorname{sign}_{0}^{+}\left(z_{1}-z_{2}\right)\right) \\
& \quad-\int_{\Omega}\left(A u_{1}(x)-A u_{2}(x)\right) \frac{1}{k} T_{k}^{+}\left(u_{1}(x)-u_{2}(x)+k \operatorname{sign}_{0}^{+}\left(z_{1}(x)-z_{2}(x)\right)\right) d x \\
& \leq \int_{\Omega}\left(\phi_{1}-\phi_{2}\right) \frac{1}{k} T_{k}^{+}\left(u_{1}-u_{2}+k \operatorname{sign}_{0}^{+}\left(z_{1}-z_{2}\right)\right) \leq \int_{\Omega}\left(\phi_{1}(x)-\phi_{2}(x)\right)^{+} d x .
\end{aligned}
$$

Let us write $u=u_{1}-u_{2}$ and $z=\operatorname{sign}_{0}^{+}\left(z_{1}-z_{2}\right)$, then, by the monotonicity proved in Lemma 2.6,

$$
\begin{aligned}
\lim _{k \rightarrow 0} & \int_{\Omega}\left(A u_{1}(x)-A u_{2}(x)\right) \frac{1}{k} T_{k}^{+}\left(u_{1}(x)-u_{2}(x)+k \operatorname{sign}_{0}^{+}\left(z_{1}(x)-z_{2}(x)\right)\right) d x \\
& =\lim _{k \rightarrow 0} \int_{\Omega} A u(x) \frac{1}{k} T_{k}^{+}(u(x)+k z(x)) d x \\
& =\lim _{k \rightarrow 0} \int_{\Omega} A(u+k z)(x) \frac{1}{k} T_{k}^{+}(u(x)+k z(x)) d x \leq 0 .
\end{aligned}
$$

Therefore, taking limit as $k$ goes to 0 in (3.1), we obtain

$$
\int_{\Omega}\left(z_{1}-z_{2}\right)^{+} \leq \int_{\Omega}\left(\phi_{1}-\phi_{2}\right)^{+} .
$$


Let us now suppose that $\phi_{1} \leq \phi_{2}, \phi_{1} \neq \phi_{2}$. By the previous calculations we know that $z_{1} \leq z_{2}$. Since

$$
\int_{\Omega} z_{1} \leq \int_{\Omega} \phi_{1}<\int_{\Omega} \phi_{2} \leq \int_{\Omega} z_{2}
$$

$z_{1} \neq z_{2}$. Going back to (3.1), if $u=u_{1}-u_{2}$, we get

$$
-\int_{\Omega} A u(x) T_{k}^{+}(u(x)) d x=0
$$

and therefore,

$$
-\int_{\Omega} A u(x) u^{+}(x) d x=0 .
$$

Consequently, by Lemma 2.6, there exists a null set $C \subset \Omega \times \Omega$ such that

$$
J(x-y)\left(u^{+}(y)-u^{+}(x)\right)(u(y)-u(x))=0 \quad \text { for all }(x, y) \in \Omega \times \Omega \backslash C .
$$

Let $B$ a null subset of $\Omega$ such that if $x \notin B$, the section $C_{x}=\{y \in \Omega:(x, y) \in C\}$ is null. Let $x \notin B$, if $u(x)>0$ then, since there exists $r_{0}>0$ such that $J(z)>0$ for every $z$ such that $|z| \leq r_{0}$, by a compactness argument and having in mind (3.2), it is easy to see that $u(y)=u(x)>0$ for all $y \notin C_{x}$. Therefore $u_{1}(y)>u_{2}(y)$ for all $y \notin C_{x}$ in $\Omega$ and consequently $z_{1}(y) \geq z_{2}(y)$ a.e. in $\Omega$ which contradicts that $z_{1} \leq z_{2}$, $z_{1} \neq z_{2}$.

Let us now prove (ii). As $\left(u_{i}, z_{i}\right)$ are solutions of $\left(S_{\phi}^{\gamma}\right)$ we have that

$$
-\left(A u_{1}(x)-A u_{2}(x)\right)+z_{1}(x)-z_{2}(x)=0 .
$$

Now, by (i), $z_{1}=z_{2}$, a.e. Consequently,

$$
0=-\left(A u_{1}(x)-A u_{2}(x)\right)=-A\left(u_{1}-u_{2}\right)(x) .
$$

Therefore, multiplying the above equation by $u=u_{1}-u_{2}$ and integrating we obtain

$$
\frac{1}{2} \int_{\Omega} \int_{\Omega} J(x-y)(u(y)-u(x))^{2} d y d x=0 .
$$

From here, by (2.2), $u$ is constant a.e. in $\Omega$.

In particular we have the following result.

Corollary 3.3. Let $k>0$ and $u \in L^{2}(\Omega)$ such that

$$
k u-A u \geq 0 \text { a.e. in } \Omega,
$$

then $u \geq 0$ a.e. in $\Omega$.

Proof. Since $(u, k u)$ is a supersolution of $\left(S_{0}^{\gamma}\right)$, where $\gamma(r)=k r$, and $(0,0)$ is a subsolution of $\left(S_{0}^{\gamma}\right)$, by Theorem 3.2, the result follows.

To study the existence of solutions of problem $\left(S_{\phi}^{\gamma}\right)$ we start with the following two lemmas.

Lemma 3.4. Assume $\gamma: \mathbb{R} \rightarrow \mathbb{R}$ is a nondecreasing Lipschitz continuous function with $\gamma(0)=0$ and $\gamma_{-}<\gamma_{+}$. Let $\phi \in C(\bar{\Omega})$ such that $\gamma_{-}<\phi<\gamma_{+}$. Then, there exists a solution $(u, \gamma(u))$ of problem $\left(S_{\phi}^{\gamma}\right)$. Moreover, $\gamma(u) \ll \phi$. 
Proof. Since $\gamma_{-}<\phi<\gamma_{+}$and $\phi \in C(\bar{\Omega})$, we can find $c_{1} \leq c_{2}$ such that

$$
\gamma_{-}<\gamma\left(c_{1}\right) \leq \phi(x) \leq \gamma\left(c_{2}\right)<\gamma_{+} \quad \forall x \in \Omega .
$$

Since $\gamma$ is a nondecreasing Lipschitz continuous function there exists $k>0$ for which the function $s \mapsto k s-\gamma(s)$ is nondecreasing. Let us see by induction that we can find a sequence $\left\{u_{i}\right\} \subset L^{2}(\Omega)$ such that

$$
\begin{gathered}
u_{0}=c_{1}, \quad u_{i} \leq u_{i+1} \leq c_{2}, \\
k u_{i+1}-A u_{i+1}=\phi-\gamma\left(u_{i}\right)+k u_{i}, \quad \forall i \in \mathbb{N} .
\end{gathered}
$$

Since $k>0$, as a consequence of being $A$ self-adjoint, it is easy to see that $k$ does not belong to the spectrum of $A$, then there exists $u_{1} \in L^{2}(\Omega)$ such that

$$
k u_{1}-A u_{1}=\phi-\gamma\left(c_{1}\right)+k c_{1} .
$$

Then, by (3.3), we have

$$
k u_{1}-A u_{1}=\phi-\gamma\left(c_{1}\right)+k c_{1} \geq k c_{1}=k c_{1}-A c_{1} .
$$

Hence, from Corollary 3.3 we get that $u_{0}=c_{1} \leq u_{1}$. Analogously, there exists $u_{2}$ such that

$$
k u_{2}-A u_{2}=\phi-\gamma\left(u_{1}\right)+k u_{1} .
$$

Now, since $c_{1} \leq u_{1}$, we get

$$
k u_{2}-A u_{2} \geq \phi-\gamma\left(c_{1}\right)+k c_{1}=k u_{1}-A u_{1} .
$$

Again by Corollary 3.3, we get $u_{1} \leq u_{2}$, and by induction we obtain that $u_{i} \leq u_{i+1}$. On the other hand, since the function $s \mapsto k s-\gamma(s)$ is nondecreasing, $c_{1} \leq c_{2}$ and (3.3), we have

$$
k c_{2}-A c_{2} \geq \phi-\gamma\left(c_{2}\right)+k c_{2} \geq \phi-\gamma\left(c_{1}\right)+k c_{1}=k u_{1}-A u_{1} .
$$

Applying again Corollary 3.3, we get $c_{2} \geq u_{1}$, and by an inductive argument we deduce that $u_{i} \leq c_{2}$ for all $i \in \mathbb{N}$. Hence (3.4) holds. Consequently, there exists $u \in L^{\infty}(\Omega)$, such that $u(x)=\lim _{i \rightarrow+\infty} u_{i}(x)$ a.e. in $\Omega$. Taking limits in (3.4), we obtain that

$$
k u-A u=\phi-\gamma(u)+k u,
$$

and $(u, \gamma(u))$ is a solution of problem $\left(S_{\phi}^{\gamma}\right)$, that is,

$$
\gamma(u)-A u=\phi .
$$

Finally, given $p \in P_{0}$, multiplying (3.5) by $p(\gamma(u))$, and integrating in $\Omega$, we get

$$
\int_{\Omega} \gamma(u(x)) p(\gamma(u(x))) d x-\int_{\Omega} A u(x) p(\gamma(u(x))) d x=\int_{\Omega} \phi(x) p(\gamma(u(x))) d x .
$$

Now, by Lemma 2.6, the second term in the above equality is nonnegative, therefore

$$
\int_{\Omega} \gamma(u(x)) p(\gamma(u(x))) d x \leq \int_{\Omega} \phi(x) p(\gamma(u(x))) d x .
$$

By Proposition 2.1, we conclude that $\gamma(u) \ll \phi$. 
Lemma 3.5. Assume $\gamma$ is a maximal monotone graph in $\left.\left.\mathbb{R}^{2},\right]-\infty, 0\right] \subset D(\gamma)$, $0 \in \gamma(0), \gamma_{-}<\gamma_{+}$. Let $\tilde{\gamma}(s)=\gamma(s)$ if $s<0, \tilde{\gamma}(s)=0$ if $s \geq 0$. Assume $\tilde{\gamma}$ is Lipschitz continuous in $]-\infty, 0]$. Let $\phi \in C(\bar{\Omega})$ such that $\gamma_{-}<\phi<\gamma_{+}$. Then, there exists a solution $(u, z)$ of $\left(S_{\phi}^{\gamma}\right)$. Moreover, $z \ll \phi$.

Proof. If $\gamma_{-}<0$, let $c_{1}$ such that $\gamma\left(c_{1}\right)=\left\{m_{1}\right\}, \gamma_{-}<m_{1}<0$ and $m_{1} \leq \phi$. And if $\gamma_{-}=0$ let $c_{1}=m_{1}=0$. Let $\gamma_{r}, r \in \mathbb{N}$, be the Yosida approximation of $\gamma$ and let the maximal monotone graph

$$
\gamma^{r}(s)= \begin{cases}\gamma(s) & \text { if } s<0 \\ \gamma_{r}(s) & \text { if } s \geq 0\end{cases}
$$

Observe that $\gamma^{r}$ is a nondecreasing Lipschitz continuous function with $\gamma^{r}(0)=0$ and, for $r$ large enough, $\gamma_{-}=\gamma_{-}^{r}<\phi<\gamma_{+}^{r}, \gamma^{r} \leq \gamma^{r+1}$, and converges in the sense of maximal monotone graphs to $\gamma$. From the previous lemma, for each $\gamma^{r}$ we obtain a solution $\left(u_{r}, z_{r}\right)$ of $\left(S_{\phi}^{\gamma^{r}}\right)$, that is, $z_{r}=\gamma^{r}\left(u_{r}\right)$ a.e. and

$$
z_{r}-A u_{r}=\phi .
$$

Moreover, $z_{r} \ll \phi$, and consequently, $z_{r} \geq m_{1}$. Moreover, $u_{r} \geq c_{1}$. Let

$$
\hat{z}_{r}(x)= \begin{cases}z_{r}(x) & \text { if } u_{r}(x) \leq 0, \\ \gamma_{r+1}\left(u_{r}(x)\right) & \text { if } u_{r}(x)>0 .\end{cases}
$$

Then, since $\gamma_{r}$ is nondecreasing,

$$
\hat{z}_{r} \geq z_{r}
$$

and also,

$$
\hat{z}_{r} \in \gamma^{r+1}\left(u_{r}\right) \text {. }
$$

Therefore, $\left(u_{r}, \hat{z}_{r}\right)$ is a supersolution to $\left(S_{\phi}^{\gamma^{r+1}}\right)$. Using Theorem 3.2, we obtain that

$$
\hat{z}_{r} \geq z_{r+1} \text {. }
$$

Now, if $\hat{z}_{r}=z_{r}$ then

$$
z_{r} \geq z_{r+1}
$$

and if $\hat{z}_{r} \neq z_{r}$, by Theorem 3.2 ,

$$
u_{r} \geq u_{r+1} \text {. }
$$

So, there exists a monotone non increasing subsequence of $\left\{u_{r}\right\}$, denoted equal, with $u_{r} \geq \hat{c}_{1}$, or there exists a monotone non increasing subsequence of $\left\{z_{r}\right\}$, denoted equal, with $z_{r} \geq m_{1}$. In the first case, we have that

$$
u_{r} \rightarrow u \quad \text { in } L^{2}(\Omega)
$$

and also, since $z_{r} \ll \phi$,

$$
z_{r} \rightarrow z \quad \text { weakly in } L^{1}(\Omega) .
$$

And in the second case, we obtain

$$
z_{r} \rightarrow z \quad \text { in } L^{1}(\Omega)
$$

In fact, since $z_{r} \ll \phi$, we get that

$$
z_{r} \rightarrow z \quad \text { in } L^{2}(\Omega) .
$$


Now, in this second case, multiplying (3.6) by $u_{r}-u_{s}$ and integrating we get

$$
-\int_{\Omega} A u_{r}\left(u_{r}-u_{s}\right)=\int_{\Omega} \phi\left(u_{r}-u_{s}\right)-\int_{\Omega} z_{r}\left(u_{r}-u_{s}\right) .
$$

Moreover,

$$
-\int_{\Omega} A u_{s}\left(u_{r}-u_{s}\right)=\int_{\Omega} \phi\left(u_{r}-u_{s}\right)-\int_{\Omega} z_{s}\left(u_{r}-u_{s}\right) .
$$

Hence, since $\int_{\Omega} z_{r}=\int_{\Omega} z_{s}$,

$$
\begin{aligned}
& -\int_{\Omega} A\left(u_{r}-u_{s}\right)\left(u_{r}-u_{s}\right)=-\int_{\Omega}\left(z_{r}-z_{s}\right)\left(u_{r}-u_{s}\right) \\
& =-\int_{\Omega}\left(z_{r}-z_{s}\right)\left(u_{r}-\frac{1}{|\Omega|} \int_{\Omega} u_{r}-\left(u_{s}-\frac{1}{|\Omega|} \int_{\Omega} u_{s}\right)\right)
\end{aligned}
$$

and, by Proposition 2.2,

$$
\beta_{1}\left\|\left(u_{r}-\frac{1}{|\Omega|} \int_{\Omega} u_{r}\right)-\left(u_{s}-\frac{1}{|\Omega|} \int_{\Omega} u_{s}\right)\right\|_{L^{2}(\Omega)} \leq\left\|z_{r}-z_{s}\right\|_{L^{2}(\Omega)} .
$$

From (3.8) we get,

$$
u_{r}-\frac{1}{|\Omega|} \int_{\Omega} u_{r} \rightarrow w \quad \text { in } L^{2}(\Omega)
$$

Let us see that $\left\{\frac{1}{|\Omega|} \int_{\Omega} u_{r}\right\}$ is bounded. If not, we can assume, passing to a subsequence if necessary, that it converges to $-\infty$. Then, $u_{r} \rightarrow-\infty$ a.e. in $\Omega$. Since $z_{r} \in \gamma^{r}\left(u_{r}\right), \gamma^{r} \rightarrow \gamma$ and (3.7), $z=\gamma_{-}$a.e. in $\Omega$. Consequently, $\int_{\Omega} \phi=\int_{\Omega} z=|\Omega| \gamma_{-}$ which contradicts that $\phi>\gamma_{-}$. Thus, $\left\{\frac{1}{|\Omega|} \int_{\Omega} u_{r}\right\}$ is bounded and we have that there exists a subsequence of $\left\{u_{r}\right\}$, denoted equal, such that

$$
u_{r} \rightarrow u \quad \text { in } L^{2}(\Omega) \text {. }
$$

Therefore, in both cases, $z \in \gamma(u)$ a.e. in $\Omega, z \ll \phi$, and, taking limit in

$$
z_{r}-A u_{r} \ni \phi
$$

we obtain

$$
z-A u \ni \phi
$$

which concludes the proof.

With this lemma in mind we proceed to extend the result for general monotone graphs.

Theorem 3.6. Assume $\gamma$ is a maximal monotone graph in $\mathbb{R}^{2}, 0 \in \gamma(0)$ and $\gamma_{-}<\gamma_{+}$. Let $\phi \in C(\bar{\Omega})$ such that $\gamma_{-}<\phi<\gamma_{+}$. Then, there exists a solution $(u, z)$ of $\left(S_{\phi}^{\gamma}\right)$. Moreover, $z \ll \phi$.

Proof. Let $\gamma_{r}, r \in \mathbb{N}$, be the Yosida approximation of $\gamma$ and let the maximal monotone graph

$$
\gamma^{r}(s)= \begin{cases}\gamma(s) & \text { if } s>0 \\ \gamma_{r}(s) & \text { if } s \leq 0\end{cases}
$$


Observe that $\gamma^{r}$ satisfies the hypothesis of Lemma 3.5, $\gamma_{-}^{r}<\phi<\gamma_{+}^{r}$ for $r$ large enough, $\gamma^{r} \geq \gamma^{r+1}$ and converges in the sense of maximal monotone graphs to $\gamma$. From the previous lemma, for each $\gamma^{r}$ we obtain a solution $\left(u_{r}, z_{r}\right)$ of $\left(S_{\phi}^{\gamma^{r}}\right), z_{r} \ll \phi$. Now, we can proceed similarly to the previous lemma passing to the limit to conclude.

The natural space to study the problem $P_{\gamma}^{J}\left(z_{0}\right)$ from the point of view of Nonlinear Semigroup Theory is $L^{1}(\Omega)$. In this space we define the following operator,

$B^{\gamma}:=\left\{(z, \hat{z}) \in L^{1}(\Omega) \times L^{1}(\Omega): \exists u \in L^{2}(\Omega)\right.$ such that $(u, z)$ is a solution of $\left.\left(S_{z+\hat{z}}^{\gamma}\right)\right\}$, in other words, $\hat{z} \in B^{\gamma}(z)$ if and only if there exists $u \in L^{2}(\Omega)$ such that $z(x) \in$ $\gamma(u(x))$ a.e. in $\Omega$, and

$$
-\int_{\Omega} J(x-y)(u(y)-u(x)) d y=\hat{z}(x), \quad \text { a.e. } x \in \Omega .
$$

The operator $B^{\gamma}$ allows us to rewrite $P_{\gamma}^{J}\left(z_{0}\right)$ as the following abstract Cauchy problem in $L^{1}(\Omega)$,

$$
\left\{\begin{array}{l}
z^{\prime}(t)+B^{\gamma}(z(t)) \ni 0 \quad t \in(0, T) \\
z(0)=z_{0} .
\end{array}\right.
$$

A direct consequence of Theorems 3.2 and 3.6 is the following result.

Corollary 3.7. Assume $\gamma$ is a maximal monotone graph in $\mathbb{R}^{2}, 0 \in \gamma(0)$. Then, the operator $B^{\gamma}$ is T-accretive in $L^{1}(\Omega)$ and satisfies

$$
\left\{\phi \in C(\bar{\Omega}): \gamma_{-}<\phi<\gamma_{+}\right\} \subset \operatorname{Ran}\left(I+B^{\gamma}\right) .
$$

The following theorem is a consequence of the above result.

Theorem 3.8. Let $T>0$ and $z_{i 0} \in L^{1}(\Omega), i=1,2$. Let $z_{i}$ be a solution in $[0, T]$ of $P_{\gamma}^{J}\left(z_{i 0}\right), i=1,2$. Then

$$
\int_{\Omega}\left(z_{1}(t)-z_{2}(t)\right)^{+} \leq \int_{\Omega}\left(z_{10}-z_{20}\right)^{+}
$$

for almost every $t \in] 0, T[$.

Proof. Let $\left(u_{i}(t), z_{i}(t)\right)$ be solutions of $P_{\gamma}^{J}\left(z_{0 i}\right), i=1,2$. Then, since they are strong solutions of (3.10) and $A$ is $T$-accretive, (3.11) follows from the Nonlinear Semigroup Theory ([7]).

In the next result we characterize ${\overline{D\left(B^{\gamma}\right)}}^{L^{1}(\Omega)}$.

Theorem 3.9. Assume $\gamma$ is a maximal monotone graph in $\mathbb{R}^{2}$. Then, we have

$$
{\overline{D\left(B^{\gamma}\right)}}^{L^{1}(\Omega)}=\left\{z \in L^{1}(\Omega): \gamma_{-} \leq z \leq \gamma_{+}\right\} .
$$

Proof. It is obvious that

$$
{\overline{D\left(B^{\gamma}\right)}}^{L^{1}(\Omega)} \subset\left\{z \in L^{1}(\Omega): \gamma_{-} \leq z \leq \gamma_{+}\right\} .
$$

To obtain the another inclusion, it is enough to take $\phi \in C(\bar{\Omega})$, satisfying $\gamma_{-}<\phi<$ $\gamma_{+}$, and to prove that $\phi \in{\overline{D\left(B^{\gamma}\right)}}^{L^{1}(\Omega)}$. Let $a, b \in \mathbb{R}$ such that $\gamma_{-}<a<\phi<b<\gamma_{+}$. 
Now, by Theorem 3.6, for any $n \in \mathbb{N}$, there exists $v_{n}:=\left(I+\frac{1}{n} B^{\gamma}\right)^{-1} \phi \in D\left(B^{\gamma}\right)$. Then, $\left(v_{n}, n\left(\phi-v_{n}\right)\right) \in B^{\gamma}$, thus there exists $u_{n} \in L^{2}(\Omega)$ such that $v_{n} \in \gamma\left(u_{n}\right)$ a.e. in $\Omega$ and

$$
v_{n}(x)-\frac{1}{n} \int_{\Omega} J(x-y)\left(u_{n}(y)-u_{n}(x)\right) d y=\phi(x) \quad \forall x \in \Omega .
$$

Moreover, $v_{n} \ll \phi$. Then,

$$
-\infty<\inf \gamma^{-1}(a) \leq u_{n} \leq \sup \gamma^{-1}(b)<+\infty .
$$

Hence, from (3.12) and (3.13) it follows that $v_{n} \rightarrow \phi$ in $L^{1}(\Omega)$.

As a consequence of the above results we have the following theorem concerning mild solutions (see [7]).

Theorem 3.10. Assume $\gamma$ is a maximal monotone graph in $\mathbb{R}^{2}$. Let $T>0$ and let $z_{0} \in L^{1}(\Omega)$ satisfying $\gamma_{-} \leq z_{0} \leq \gamma_{+}$. Then, there exists a unique mild solution of (3.10). Moreover $z \ll z_{0}$.

Proof. For $n \in \mathbb{N}$, let $\varepsilon=T / n$, and consider a subdivision $t_{0}=0<t_{1}<\cdots<t_{n-1}<$ $T=t_{n}$ with $t_{i}-t_{i-1}=\varepsilon$. Let $z_{0}^{\varepsilon} \in C(\bar{\Omega})$ with

$$
\gamma_{-}<z_{0}^{\varepsilon}<\gamma_{+}
$$

and

$$
\left\|z_{0}^{\varepsilon}-z_{0}\right\|_{L^{1}(\Omega)} \leq \varepsilon
$$

By Theorem 3.6, for $n$ large enough, there exists a solution $\left(u_{i}^{\varepsilon}, z_{i}^{\varepsilon}\right)$ of

$$
\gamma\left(u_{i}^{\varepsilon}\right)-\varepsilon A u_{i}^{\varepsilon} \ni z_{i-1}^{\varepsilon}
$$

for $i=1, \ldots, n$, with

$$
z_{i}^{\varepsilon} \ll z_{i-1}^{\varepsilon} .
$$

That is, there exists a unique solution $z_{i}^{\varepsilon} \in L^{1}(\Omega)$ of the time discretized scheme associated with (3.10),

$$
z_{i}^{\varepsilon}+\varepsilon B^{\gamma} z_{i}^{\varepsilon} \ni \varepsilon z_{i-1}^{\varepsilon}, \quad \text { for } i=1, \ldots, n .
$$

Therefore, if we define $z_{\varepsilon}(t)$ by

$$
\left\{\begin{array}{l}
z_{\varepsilon}(0)=z_{0}^{\varepsilon}, \\
\left.\left.z_{\varepsilon}(t)=z_{i}^{\varepsilon}, \quad \text { for } t \in\right] t_{i-1}, t_{i}\right], \quad i=1, \ldots, n,
\end{array}\right.
$$

it is an $\varepsilon$-approximate solution of problem (3.10).

By using now the Nonlinear Semigroup Theory (see [5], [7], [14]), on account of Corollary 3.7 and Theorem 3.9, problem (3.10) has a unique mild-solution $z(t) \in$ $C\left([0, T]: L^{1}(\Omega)\right)$, obtained as $z(t)=L^{1}(\Omega)-\lim _{\varepsilon \rightarrow 0} z_{\varepsilon}(t)$ uniformly for $t \in[0, T]$. Finally, from (3.15) we get $z \ll z_{0}$.

By Crandall-Liggett's Theorem, [14], the mild solution obtained above is given by the well-known exponential formula,

$$
e^{-t B^{\gamma}} z_{0}=\lim _{n \rightarrow \infty}\left(I+\frac{t}{n} B^{\gamma}\right)^{-n} z_{0} .
$$


The nonlinear contraction semigroup $e^{-t B^{\gamma}}$ generated by the operator $-B^{\gamma}$ will be denoted in the sequel by $(S(t))_{t \geq 0}$.

In principle, it is not clear how these mild solutions have to be interpreted respect to $P_{\gamma}^{J}\left(z_{0}\right)$. In the next section we will see that they coincide with the solutions defined in the Introduction.

\section{Existence of SOlutions}

In this section we prove that the mild solution of (3.10) is in fact a solution in the sense of Definition 1.1. of problem $P_{\gamma}^{J}\left(z_{0}\right)$.

Theorem 4.1. Let $z_{0} \in L^{1}(\Omega)$ such that $\gamma_{-} \leq z_{0} \leq \gamma_{+}, \gamma_{-}<\frac{1}{|\Omega|} \int_{\Omega} z_{0}<\gamma_{+}$and $\int_{\Omega} j_{\gamma}^{*}\left(z_{0}\right)<+\infty$. Then, there exists a unique solution to $P_{\gamma}^{J}\left(z_{0}\right)$ in $[0, T]$ for every $T>0$. Moreover, $z \ll z_{0}$.

Proof. We divide the proof in three steps.

Step 1. First, let us suppose that

$$
\begin{aligned}
& \text { there exist } c_{1}, c_{2} \text { such that } c_{1} \leq c_{2}, m_{1} \in \gamma\left(c_{1}\right), m_{2} \in \gamma\left(c_{2}\right) \\
& \text { and } \gamma_{-}<m_{1} \leq z_{0} \leq m_{2}<\gamma_{+} .
\end{aligned}
$$

Let $z(t)$ be the mild solution of (3.10) given by Theorem 3.10. We shall show that $z$ is a solution of problem $P_{\gamma}^{J}\left(z_{0}\right)$.

For $n \in \mathbb{N}$, let $\varepsilon=T / n$, and consider a subdivision $t_{0}=0<t_{1}<\cdots<t_{n-1}<$ $T=t_{n}$ with $t_{i}-t_{i-1}=\varepsilon$. Then, it follows that

$$
z(t)=L^{1}(\Omega)-\lim _{\varepsilon} z_{\varepsilon}(t) \quad \text { uniformly for } t \in[0, T],
$$

where $z_{\varepsilon}(t)$ is given, for $\varepsilon$ small enough, by

$$
\begin{cases}z_{\varepsilon}(t)=z_{0} & \text { for } t \in]-\infty, 0], \\ z_{\varepsilon}(t)=z_{i}^{n}, & \text { for } \left.t \in] t_{i-1}, t_{i}\right], \quad i=1, \ldots, n,\end{cases}
$$

where $\left(u_{i}^{n}, z_{i}^{n}\right) \in L^{2}(\Omega) \times L^{1}(\Omega)$ is the solution of

$$
-A u_{i}^{n}+\frac{z_{i}^{n}-z_{i-1}^{n}}{\varepsilon}=0, \quad i=1,2, \ldots, n .
$$

Moreover, $z_{i}^{n} \ll z_{0}$. Hence $\gamma_{-}<m_{1} \leq z_{i}^{n} \leq m_{2}<\gamma_{+}$and consequently,

$$
\inf \gamma^{-1}\left(m_{1}\right) \leq u_{i}^{n} \leq \sup \gamma^{-1}\left(m_{2}\right) \text {. }
$$

Therefore, if we write $\left.\left.u_{\varepsilon}(t)=u_{i}^{n}, t \in\right] t_{i-1}, t_{i}\right], i=1, \ldots, n$, we can suppose that

$$
u_{\varepsilon} \rightarrow u \quad \text { weakly in } L^{2}\left(0, T ; L^{2}(\Omega)\right) \text { as } \varepsilon \rightarrow 0^{+} .
$$

Since $z_{\varepsilon} \in \gamma\left(u_{\varepsilon}\right)$ a.e. in $Q_{T}, z_{\varepsilon} \rightarrow z$ in $L^{1}\left(Q_{T}\right)$, having in mind (4.5), we obtain that $z \in \gamma(u)$ a.e. in $Q_{T}$. On the other hand, from (4.4),

$$
\frac{z_{\varepsilon}(t)-z_{\varepsilon}(t-\varepsilon)}{\varepsilon} \rightarrow z_{t} \quad \text { weakly in } L^{2}\left(0, T ; L^{2}(\Omega)\right) \text { as } \varepsilon \rightarrow 0^{+} .
$$

Step 2. Let now $z_{0} \in L^{1}(\Omega)$ such that $\gamma_{-} \leq z_{0} \leq \gamma_{+}, \gamma_{-}|\Omega|<\int_{\Omega} z_{0}<\gamma_{-}|\Omega|$, $\int_{\Omega} j_{\gamma}^{*}\left(z_{0}\right)<+\infty$, and

there exists $c_{1}$ and $m_{1} \in \gamma\left(c_{1}\right)$ with $\gamma_{-}<m_{1} \leq z_{0}$ and (4.1) is not satisfied. 
Let $z_{0 n} \in L^{\infty}(\Omega)$,

$$
z_{0 n} \nearrow z_{0} \text { as } n \text { goes to }+\infty
$$

such that $\int_{\Omega} z_{0 n}<\int_{\Omega} z_{0 n+1}$ and $z_{0 n} \leq m_{2}(n)<\gamma_{+}, m_{2}(n) \in \gamma\left(c_{2}(n)\right)$ for some $c_{2}(n)$. By Step 1, there exists a solution $z_{n}$ of problem $P_{\gamma}^{J}\left(z_{0 n}\right)$, which is the mild solution of (3.10) with initial datum $z_{0 n}$ and satisfies $z_{n} \ll z_{0 n}$. It is obvious that

$$
\lim _{n \rightarrow \infty} z_{n}=z \quad \text { in } C\left([0, T]: L^{1}(\Omega)\right),
$$

being $z$ the mild solution of (3.10) with initial datum $z_{0}$, moreover $z \ll z_{0}$. Next we prove that $z$ is the solution of $P_{\gamma}^{J}\left(z_{0}\right)$.

Since $z_{n}$ is a solution of problem $P_{\gamma}^{J}\left(z_{0 n}\right)$, there exists $u_{n} \in L^{2}\left(0, T, L^{2}(\Omega)\right), z_{n} \in$ $\gamma\left(u_{n}\right)$ a.e. in $\left.\Omega \times\right] 0, T[$, such that

$$
\left(z_{n}\right)_{t}-A u_{n}=0 .
$$

Moreover, we can suppose that (see Theorem 3.2)

$$
u_{n} \text { is non decreasing in } n \text {. }
$$

Multiplying (4.7) by $u_{n}$, we obtain

$$
\frac{d}{d t} \int_{\Omega}\left(\int_{0}^{z_{n}(t)}\left(\gamma^{-1}\right)^{0}(s) d s\right)=\int_{\Omega} A u_{n}(t) u_{n}(t) d t
$$

in $\mathcal{D}^{\prime}(] 0, T[)$. Indeed, since $u_{n}(t) \in \gamma^{-1}\left(z_{n}(t)\right)=\partial j_{\gamma}^{*}\left(z_{n}(t)\right)$,

$$
\left(z_{n}(t+\tau)-z_{n}(t)\right) u_{n}(t) \leq \int_{z_{n}(t)}^{z_{n}(t+\tau)}\left(\gamma^{-1}\right)^{0}(s) d s \text { for all } \tau .
$$

Consequently,

$$
\int_{\Omega}\left(z_{n}\right)_{t}(t) u_{n}(t)=\frac{d}{d t} \int_{\Omega}\left(\int_{0}^{z_{n}(t)}\left(\gamma^{-1}\right)^{0}(s) d s\right)
$$

and (4.9) holds.

Integrating now (4.9) between 0 and $T$ we get

$$
-\int_{0}^{T} \int_{\Omega} A u_{n}(t) u_{n}(t) d t \leq \int_{\Omega} j_{\gamma}^{*}\left(z_{0}\right)
$$

Let us see that $\left\{u_{n}\right\}$ is bounded in $L^{2}\left(Q_{T}\right)$. In the case $\gamma_{+}=+\infty$, let

$$
M=\sup _{t \in[0, T]} \int_{\Omega} z^{+}(t)+1 .
$$

Then, there exists $n_{0} \in \mathbb{N}$ such that

$$
\sup _{t \in[0, T]} \int_{\Omega}\left(z_{n}\right)^{+}(t)<M, \quad \forall n \geq n_{0} .
$$

In the case $\gamma_{+}<+\infty$, since we have conservation of mass, there exists $M \in \mathbb{R}$ and $n_{0} \in \mathbb{N}$ such that, for all $n \geq n_{0}$,

$$
\sup _{t \in[0, T]} \int_{\Omega} z_{n}(t)<M<\gamma_{+}|\Omega|,
$$


moreover, since $z_{n} \ll z_{0}$, we have that $z_{n} \geq m_{1}$ and, since it is not difficult to see that $\left|m_{1}\right|<\frac{\gamma_{+}|\Omega|-M}{4|\Omega|}$, we have

$$
\sup _{t \in[0, T]} \int_{\left\{x \in \Omega: z_{n}(t)(x)<-4\left(m_{1}^{2}+1\right)|\Omega| /\left(\gamma_{+}|\Omega|-M\right)\right\}}\left|z_{n}(t)\right|<\frac{\gamma_{+}|\Omega|-M}{4}, \quad \forall n \in \mathbb{N} .
$$

Therefore, in both cases, by Lemma 2.5, there exists $C>0$ such that

$$
\left\|\left(u_{n}(t)\right)^{+}\right\|_{L^{2}(\Omega)} \leq C\left(\left(-\int_{\Omega} A\left(u_{n}(t)\right)^{+}\left(u_{n}(t)\right)^{+}\right)^{1 / 2}+1\right), \quad \forall t \in[0, T] .
$$

Hence, by (4.10), since $u_{n}$ is non decreasing in $n,\left\{u_{n}\right\}$ is bounded in $L^{2}\left(Q_{T}\right)$.

Passing to a subsequence if necessary, we can assume

$$
u_{n} \rightarrow u \quad \text { weakly in } L^{2}\left(0, T ; L^{2}(\Omega)\right) \text { as } n \rightarrow+\infty,
$$

and, by (4.8),

$$
u_{n} \rightarrow u \quad \text { in } L^{2}\left(0, T ; L^{2}(\Omega)\right) \text { as } n \rightarrow+\infty
$$

Consequently,

$$
z \in \gamma(u) \text { a.e. in } Q_{T} \text {. }
$$

Since also $\left\{A u_{n}\right\}$ is bounded in $L^{2}\left(Q_{T}\right)$, passing to the limit in (4.7) we get

$$
z_{t}-A u=0 .
$$

Step 3. Let now $z_{0} \in L^{1}(\Omega), \gamma_{-} \leq z_{0} \leq \gamma_{+}$and $\gamma_{-}|\Omega|<\int_{\Omega} z_{0}<\gamma_{-}|\Omega|, \int_{\Omega} j_{\gamma}^{*}\left(z_{0}\right)<$ $+\infty$ such that (4.6) is not satisfied. Let $z_{0 n} \in L^{\infty}(\Omega)$,

$$
z_{0 n} \searrow z_{0} \quad \text { as } n \text { goes to }+\infty
$$

such that $\int_{\Omega} z_{0 n}>\int_{\Omega} z_{0 n+1}$ and $z_{0 n} \geq m_{1}(n)>\gamma_{-}, m_{1}(n) \in \gamma\left(c_{1}(n)\right)$ for some $c_{1}(n)$. By Step 2, there exist a solution $z_{n}$ of problem $P_{\gamma}^{J}\left(z_{0 n}\right)$, which is the mild solution of (3.10) with initial datum $z_{0 n}$ and satisfies $z_{n} \ll z_{0}$. It is obvious that

$$
\lim _{n \rightarrow \infty} z_{n}=z \quad \text { in } C\left([0, T]: L^{1}(\Omega)\right),
$$

being $z$ the mild solution of (3.10) with initial datum $z_{0}$. Moreover $z \ll z_{0}$. We shall see that $z$ is the solution of $P_{\gamma}^{J}\left(z_{0}\right)$. The proof is similar to the above step and we only need to take care in the proof of the boundedness of $\left\{u_{n}\right\}$ in $L^{2}\left(Q_{T}\right)$. To this end we need a formula like (4.11) for $u_{n}^{-}$, that is, we need to prove that there exists $C>0$ such that

$$
\left\|\left(u_{n}(t)\right)^{-}\right\|_{L^{2}(\Omega)} \leq C\left(\left(-\int_{\Omega} A\left(u_{n}(t)\right)^{-}\left(u_{n}(t)\right)^{-}\right)^{1 / 2}+1\right), \quad \forall t \in[0, T] .
$$

Let us consider first that $\gamma_{-}=-\infty$, and let

$$
M=\sup _{t \in[0, T]} \int_{\Omega} z^{-}(t)+1
$$

Then, there exists $n_{0} \in \mathbb{N}$ such that

$$
\sup _{t \in[0, T]} \int_{\Omega}\left(z_{n}\right)^{-}(t)<M, \quad \forall n \geq n_{0} .
$$


In the case $\gamma_{-}>-\infty$, there exists $M \in \mathbb{R}, h>0$ and $n_{0} \in \mathbb{N}$ such that, for all $n \geq n_{0}$,

$$
\inf _{t \in[0, T]} \int_{\Omega} z_{n}(t)>M>\gamma_{-}|\Omega|
$$

and

$$
\sup _{t \in[0, T]} \int_{\left\{x \in \Omega: z_{n}(t)(x)>h\right\}} z_{n}(t)<\frac{M-\gamma_{-}|\Omega|}{4} .
$$

Formula (4.14) is straightforward and (4.15) follows from (4.12). Indeed, by (4.12), there exists $n_{0} \in \mathbb{N}, \delta>0$ and $h>0$ such that, for all $n \geq n_{0}$ and for all $t \in[0, T]$,

$$
\int_{E}\left|z_{n}(t)\right|<\frac{M-\gamma_{-}|\Omega|}{4}, \quad \forall E \subset \Omega,|E|<\delta
$$

and we can take $h$ satisfying

$$
\left|\left\{x \in \Omega: z_{n}(t)(x)>h\right\}\right|<\delta \text {. }
$$

Therefore, in both cases, by Lemma 2.5, (4.13) is proved.

Uniqueness of solutions follows from Theorem 3.8.

\section{Asymptotic Behaviour}

In this section we study the asymptotic behaviour of the solutions to $P_{\gamma}^{J}\left(z_{0}\right)$. Note that since the solution preserves the total mass it is natural to expect that solutions to our diffusion problem converge to the mean value of the initial condition as $t \rightarrow \infty$. We shall see that this is the case, for instance, when $\gamma$ is a continuous function, nevertheless this fails when $\gamma$ has jumps.

Let us introduce the $\omega$-limit set for a given initial condition $z_{0}$,

$$
\omega\left(z_{0}\right)=\left\{w \in L^{1}(\Omega): \exists t_{n} \rightarrow \infty \text { with } S\left(t_{n}\right) z_{0} \rightarrow w, \text { strongly in } L^{1}(\Omega)\right\}
$$

and the weak $\omega$-limit set

$$
\omega_{\sigma}\left(z_{0}\right)=\left\{w \in L^{1}(\Omega): \exists t_{n} \rightarrow \infty \text { with } S\left(t_{n}\right) z_{0} \rightarrow w, \text { weakly in } L^{1}(\Omega)\right\} .
$$

Since $S(t) z_{0} \ll z_{0}, \omega_{\sigma}\left(z_{0}\right) \neq \emptyset$ always. Moreover since $S(t)$ preserves the total mass, for all $w \in \omega_{\sigma}\left(z_{0}\right)$,

$$
\int_{\Omega} w=\int_{\Omega} z_{0}
$$

We denote by $F$ the set of fixed points of the semigroup $(S(t))$, that is,

$$
F=\left\{w \in{\overline{D\left(B^{\gamma}\right)}}^{L^{1}(\Omega)}: S(t) w=w \quad \forall t \geq 0\right\} .
$$

It is easy to see that

$$
F=\left\{w \in L^{1}(\Omega): \exists k \in D(\gamma) \text { such that } w \in \gamma(k)\right\} .
$$

Theorem 5.1. Let $z_{0} \in L^{1}(\Omega)$ such that $\gamma_{-} \leq z_{0} \leq \gamma_{+}, \gamma_{-}<\frac{1}{|\Omega|} \int_{\Omega} z_{0}<\gamma_{+}$and $\int_{\Omega} j_{\gamma}^{*}\left(z_{0}\right)<+\infty$. Then, $\omega_{\sigma}\left(z_{0}\right) \subset F$. Moreover, if $\omega\left(z_{0}\right) \neq \emptyset$, then $\omega\left(z_{0}\right)$ consists of a unique $w \in F$, and consequently,

$$
\lim _{t \rightarrow \infty} S(t) z_{0}=w \quad \text { strongly in } L^{1}(\Omega)
$$


Proof. Along this proof we denote by $z(t)=S(t) z_{0}$ the solution to problem $P_{\gamma}^{J}\left(z_{0}\right)$ and $u(t)$ the corresponding function that appears in Definition 1.1.

Multiplying the equation in $P_{\gamma}^{J}\left(z_{0}\right)$ by $u(t)$ and integrating, we deduce

$$
-\int_{0}^{+\infty} \int_{\Omega} A u(t) u(t) d t \leq \int_{\Omega} j_{\gamma}^{*}\left(z_{0}\right) .
$$

Therefore, thanks to (2.2), we obtain that there exists a constant $C$ such that

$$
\int_{0}^{+\infty} \int_{\Omega}\left|u(t)-\frac{1}{|\Omega|} \int_{\Omega} u(t)\right|^{2} d t \leq C .
$$

Let $w \in \omega_{\sigma}\left(z_{0}\right)$, then there exists a sequence $t_{n} \rightarrow+\infty$ such that $S\left(t_{n}\right) z_{0} \rightarrow w$. By (5.3), we have

Take $s_{n} \rightarrow 0$ such that

$$
\alpha_{n}:=\int_{t_{n}}^{+\infty} \int_{\Omega}\left|u(t)-\frac{1}{|\Omega|} \int_{\Omega} u(t)\right|^{2} d t \rightarrow 0 .
$$

$$
\lim _{n \rightarrow \infty} \frac{\alpha_{n}}{s_{n}}=0 .
$$

By contradiction it is easy to see that there exists $\bar{t}_{n} \in\left[t_{n}, t_{n}+\frac{C}{s_{n}}\right]$ such that

$$
\int_{\Omega}\left|u\left(\bar{t}_{n}\right)-\frac{1}{|\Omega|} \int_{\Omega} u\left(\bar{t}_{n}\right)\right|^{2} \leq s_{n}
$$

and consequently,

$$
\int_{\Omega}\left|u\left(\bar{t}_{n}\right)-\frac{1}{|\Omega|} \int_{\Omega} u\left(\bar{t}_{n}\right)\right|^{2} \rightarrow 0 .
$$

Let us prove that

$$
\frac{1}{|\Omega|} \int_{\Omega} u\left(\bar{t}_{n}\right)
$$

is bounded. In fact, assume there exists a subsequence (still denoted by $\bar{t}_{n}$ ) such that

$$
\frac{1}{|\Omega|} \int_{\Omega} u\left(\bar{t}_{n}\right) \rightarrow+\infty
$$

By (5.5) we get that $u\left(\bar{t}_{n}\right) \rightarrow+\infty$ a.e. Since $z\left(\bar{t}_{n}\right) \in \gamma\left(u\left(\bar{t}_{n}\right)\right)$, then $z\left(\bar{t}_{n}\right) \rightarrow \gamma_{+}$ a.e. Moreover, as $z\left(\bar{t}_{n}\right) \ll z_{0}$ and $\gamma^{+} \geq 0$, we can deduce that $\lim _{n \rightarrow \infty} z\left(\bar{t}_{n}\right)=$ $\lim _{n \rightarrow \infty} z\left(\bar{t}_{n}\right)^{+}$weakly in $L^{1}(\Omega)$. Hence, applying Fatou's Lemma, we get

$$
\int_{\Omega} z_{0}=\lim _{n \rightarrow \infty} \int_{\Omega} z\left(\bar{t}_{n}\right)^{+} \geq \gamma_{+}|\Omega|
$$

a contradiction. A similar argument shows that $\frac{1}{|\Omega|} \int_{\Omega} u\left(\bar{t}_{n}\right)$ is bounded from below.

Therefore, passing to a subsequence if necessary, we may assume that

$$
\frac{1}{|\Omega|} \int_{\Omega} u\left(\bar{t}_{n}\right) \rightarrow k
$$

for some constant $k$. Using again (5.5),

$$
u\left(\bar{t}_{n}\right) \rightarrow k, \quad \text { strongly in } L^{2}(\Omega) \text { and a.e. }
$$


Since $z\left(\bar{t}_{n}\right) \ll z_{0}$, we can assume, taking a subsequence if necessary, that $z\left(\bar{t}_{n}\right) \rightarrow \hat{w}$ weakly in $L^{1}(\Omega)$. Then, from (5.6) it follows that $\hat{w} \in \gamma(k)$, and consequently $\hat{w} \in F$. Let us show now that $w=\hat{w}$. By (5.4), we have

$$
\begin{gathered}
\left\|z\left(\bar{t}_{n}\right)-z\left(t_{n}\right)\right\|=\left\|\int_{t_{n}}^{\bar{t}_{n}} z_{t}(s) d s\right\|_{L^{1}(\Omega)}=\left\|\int_{t_{n}}^{\bar{t}_{n}} A u(s) d s\right\|_{L^{1}(\Omega)} \\
\leq M\left(\bar{t}_{n}-t_{n}\right)^{1 / 2}\left(\int_{t_{n}}^{+\infty} \int_{\Omega}\left|u(s)-\frac{1}{|\Omega|} \int_{\Omega} u(s)\right|^{2} d s\right)^{1 / 2} \leq M\left(C \frac{\alpha_{n}}{s_{n}}\right)^{1 / 2} \rightarrow 0,
\end{gathered}
$$

where $M$ is a constant depending of $|\Omega|$. Therefore, taking limit, we get $z\left(\bar{t}_{n}\right)-$ $z\left(t_{n}\right) \rightarrow 0$, strongly in $L^{1}(\Omega)$, since it converges weakly to $\bar{w}-w$, it follows that $w=\bar{w}$, which is a fixed point. Finally, if $\omega\left(z_{0}\right) \neq \emptyset$, since $\omega\left(z_{0}\right) \subset \omega_{\sigma}\left(z_{0}\right) \subset F$ and $(S(t))$ is a contraction semigroup, we have that $\omega\left(z_{0}\right)=\{w\} \subset F$ and

$$
\lim _{t \rightarrow \infty} S(t) z_{0}=w \quad \text { strongly in } L^{1}(\Omega) \text {. }
$$

Remark 5.2. Note that in order to proof that $\omega\left(z_{0}\right) \neq \emptyset$, a usual tool is to show that the resolvent of $B^{\gamma}$ is compact. In our case this fails in general as the following example shows. Let $\gamma$ any maximal monotone graph with $\gamma(0)=[0,1], z_{n} \in L^{\infty}(\Omega)$, $0 \leq z_{n} \leq 1$ such that $\left\{z_{n}\right\}$ is not relatively compact in $L^{1}(\Omega)$. It is easy to check that $z_{n}=\left(I+B^{\gamma}\right)^{-1}\left(z_{n}\right)$. Hence $\left(I+B^{\gamma}\right)^{-1}$ is not a compact operator in $L^{1}(\Omega)$. On the other hand, since the nonlocal operator does not have regularizing effects, here we cannot prove regularity properties of the solutions that would help to find compactness of the orbits. Nevertheless, we shall see in the next result that when $\gamma$ is a continuous function we are able to prove that $\omega\left(z_{0}\right) \neq \emptyset$.

Let us see now some cases in which $\omega\left(z_{0}\right) \neq \emptyset$ and

$$
\lim _{t \rightarrow \infty} S(t) z_{0}=\frac{1}{|\Omega|} \int_{\Omega} z_{0} \quad \text { strongly in } L^{1}(\Omega) .
$$

Given a maximal monotone graph $\gamma$ in $\mathbb{R} \times \mathbb{R}$, we set

$$
\gamma(r+):=\inf \gamma(] r,+\infty[), \quad \gamma(r-):=\sup \gamma(]-\infty, r[)
$$

for $r \in \mathbb{R}$, where we use the conventions $\inf \emptyset=+\infty$ and $\sup \emptyset=-\infty$. It is easy to see that

$$
\gamma(r)=[\gamma(r-), \gamma(r+)] \cap \mathbb{R} \quad \text { for } \quad r \in \mathbb{R}
$$

Moreover, $\gamma(r-)=\gamma(r+)$ except at a countable set of points, which we denote by $J(\gamma)$.

Corollary 5.3. Let $z_{0} \in L^{1}(\Omega)$ such that $\gamma_{-} \leq z_{0} \leq \gamma_{+}, \gamma_{-}<\frac{1}{|\Omega|} \int_{\Omega} z_{0}<\gamma_{+}$and $\int_{\Omega} j_{\gamma}^{*}\left(z_{0}\right)<+\infty$. The following statements hold.

(1) If $\frac{1}{|\Omega|} \int_{\Omega} z_{0} \notin \gamma(J(\gamma))$ or $\frac{1}{|\Omega|} \int_{\Omega} z_{0} \in\{\gamma(k+), \gamma(k-)\}$ for some $k \in J(\gamma)$, then

$$
\lim _{t \rightarrow \infty} S(t) z_{0}=\frac{1}{|\Omega|} \int_{\Omega} z_{0} \quad \text { strongly in } L^{1}(\Omega) .
$$


(2) If $\gamma$ is a continuous function then

$$
\lim _{t \rightarrow \infty} S(t) z_{0}=\frac{1}{|\Omega|} \int_{\Omega} z_{0} \quad \text { strongly in } L^{1}(\Omega) .
$$

(3) If $\left.\frac{1}{|\Omega|} \int_{\Omega} z_{0} \in\right] \gamma(k-), \gamma(k+)[$ for some $k \in J(\gamma)$, then

$$
\omega_{\sigma}\left(z_{0}\right) \subset\left\{w \in L^{1}(\Omega): w \in[\gamma(k-), \gamma(k+)] \text { a.e., } \int_{\Omega} w=\int_{\Omega} z_{0}\right\} .
$$

and consequently, for any $w \in \omega_{\sigma}\left(z_{0}\right)$, there exists a non null set in which $w \in] \gamma(k-), \gamma(k+)[$.

Proof. (1). Along this proof we denote by $z(t)=S(t) z_{0}$ the solution to problem $P_{\gamma}^{J}\left(z_{0}\right)$ and $u(t)$ the corresponding function that appears in Definition 1.1. First, let us assume that $\frac{1}{|\Omega|} \int_{\Omega} z_{0} \notin \gamma(J(\gamma))$ and $z_{0} \in L^{\infty}(\Omega)$. Working as in the above theorem, we have that there exists a constat $k$ such that

$$
u\left(t_{n}\right) \rightarrow k, \quad \text { strongly in } L^{2}(\Omega) \text { and a.e. }
$$

Since $z\left(t_{n}\right) \ll z_{0}$, there exists a subsequence such that $z\left(t_{n}\right) \rightarrow w$ weakly in $L^{1}(\Omega)$. Now, from $z\left(t_{n}\right) \in \gamma\left(u\left(t_{n}\right)\right)$ we deduce that $w \in \gamma(k)$ and consequently, since $\frac{1}{|\Omega|} \int_{\Omega} z_{0}=\frac{1}{|\Omega|} \int_{\Omega} w, k \notin J(\gamma)$. Then, there exists $\delta>0$ such that $\gamma$ is univalued and continuous on $] k-\delta, k+\delta\left[\right.$. Hence, $w=\gamma(k)$ and $z\left(t_{n}\right) \rightarrow \gamma(k)$ a.e. Therefore, Since $z\left(t_{n}\right)$ is bounded in $L^{\infty}(\Omega), z\left(t_{n}\right) \rightarrow \gamma(k)=\frac{1}{|\Omega|} \int_{\Omega} z_{0}$ strongly in $L^{1}(\Omega)$. Then, by the above theorem we get that

$$
z(t) \rightarrow \frac{1}{|\Omega|} \int_{\Omega} z_{0}, \quad \text { as } t \rightarrow \infty .
$$

The general case $z_{0} \in L^{1}(\Omega)$ follows easily from the previous arguments using again that we deal with a contraction semigroup.

Assume now that $\frac{1}{|\Omega|} \int_{\Omega} z_{0} \in\{\gamma(k+), \gamma(k-)\}$ for some $k \in J(\gamma)$. It is easy to see that we can find $z_{0, n} \in L^{1}(\Omega)$, with $\gamma_{-} \leq z_{0, n} \leq \gamma_{+}, \gamma_{-}<\frac{1}{|\Omega|} \int_{\Omega} z_{0, n}<\gamma_{+}$and $\int_{\Omega} j_{\gamma}^{*}\left(z_{0, n}\right)<+\infty$, such that $z_{0, n} \rightarrow z_{0}$ strongly in $L^{1}(\Omega)$ and verifying $\frac{1}{|\Omega|} \int_{\Omega} z_{0, n} \notin$ $\gamma(J(\gamma))$ for all $n$. Then, by the above step, we have

$$
S(t) z_{0, n} \rightarrow \frac{1}{|\Omega|} \int_{\Omega} z_{0, n}, \quad \text { strongly in } L^{1}(\Omega),
$$

from where it follows, using again that $(S(t))$ is a contraction semigroup, that

$$
S(t) z_{0} \rightarrow \frac{1}{|\Omega|} \int_{\Omega} z_{0}, \quad \text { strongly in } L^{1}(\Omega) .
$$

Statement (2) is an obvious consequence of (1) since in this case $J(\gamma)=\emptyset$.

Finally, we prove (3). Given $w \in \omega_{\sigma}\left(z_{0}\right)$, by Theorem 5.1, there exists $k_{0} \in D(\gamma)$, such that $w \in \gamma\left(k_{0}\right)$. Then, $k_{0}=k$. In fact, if we assume, for instance, that $k_{0}<k$, then

$$
\gamma\left(k_{0}+\right) \geq \frac{1}{|\Omega|} \int_{\Omega} w=\frac{1}{|\Omega|} \int_{\Omega} z_{0}>\gamma(k-)>\gamma\left(k_{0}+\right),
$$


a contradiction. Hence, we have $w \in \gamma(k)$, and

$$
\left.\frac{1}{|\Omega|} \int_{\Omega} w=\frac{1}{|\Omega|} \int_{\Omega} z_{0} \in\right] \gamma(k-), \gamma(k+)[.
$$

Thus, $w \in[\gamma(k-), \gamma(k+)]$ a.e. and, moreover, there exists a non null set in which $w \in] \gamma(k-), \gamma(k+)[$.

Remark 5.4. An alternative proof of the fact that $\omega\left(z_{0}\right) \subset F$ is the following. Let

$$
\left.\left.\Psi: L^{1}(\Omega) \rightarrow\right]-\infty,+\infty\right]
$$

the functional defined by

$$
\Psi(z):=\left\{\begin{array}{lll}
\int_{\Omega} j_{\gamma}^{*}(z) & \text { if } & j_{\gamma}^{*}(z) \in L^{1}(\Omega), \\
+\infty & \text { if } & j_{\gamma}^{*}(z) \notin L^{1}(\Omega) .
\end{array}\right.
$$

Since $j_{\gamma}^{*}$ is continuous and convex, $\Psi$ is lower semi-continuous ([8], pag.160). Moreover, since $S(t) z_{0} \ll z_{0}$ for all $t \geq 0$, we have $\Psi\left(S(t) z_{0}\right) \leq \Psi\left(z_{0}\right)$ for all $t \geq 0$. Therefore, $\Psi$ is a lower semi-continuous Liapunov functional for $(S(t))$. Then, by the Invariance Principle of Dafermos $([15]), \Psi$ is constant on $\omega\left(z_{0}\right)$. Consequently, given $w_{0} \in \omega\left(z_{0}\right)$, if $w(t)=S(t) w_{0}$, we have $\Psi(w(t))$ is constant for all $t \geq 0$. Let $u(t)$ such that $w(t) \in \gamma(u(t))$ and $w_{t}=A(u(t))$. Working as in the proof of (4.9), we get

$$
0=\frac{d}{d t} \Psi(w(t))=\frac{d}{d t} \int_{\Omega} j_{\gamma}^{*}(w(t))=\frac{d}{d t} \int_{\Omega} j_{\gamma^{-1}}(w(t))=\int_{\Omega} A u(t) u(t) .
$$

Then, by Proposition 2.2, we obtain that

$$
u(t)=\frac{1}{|\Omega|} \int_{\Omega} u(t)
$$

Hence, $w(t) \in F$ for all $t>0$, and consequently, $w_{0} \in F$.

Acknowledgements. Part of this work was performed during a visit of JDR to Univ. de Valencia. He wants to thank for the warm hospitality and the stimulating working atmosphere found there. FA, JMM and JT are partially supported by the Spanish MEC and FEDER, project MTM2005-00620, and by project ACOMP06/196 from Generalitat Valenciana. JDR is partially supported by ANPCyT PICT 5009, UBA X066, Fundación Antorchas and CONICET (Argentina).

\section{REFERENCES}

[1] F. Andreu, N. Igbida, J. M. Mazón and J. Toledo. A degenerate elliptic-parabolic problem with nonlinear dynamical boundary conditions. To appear in Interfaces Free Bound.

[2] P. Bates and A. Chmaj. An integrodifferential model for phase transitions: stationary solutions in higher dimensions. J. Statistical Phys., 95, 1119-1139, (1999).

[3] P. Bates and A. Chmaj. A discrete convolution model for phase transitions. Arch. Rat. Mech. Anal., 150, 281-305, (1999).

[4] P. Bates, P. Fife, X. Ren and X. Wang. Travelling waves in a convolution model for phase transitions. Arch. Rat. Mech. Anal., 138, 105-136, (1997).

[5] Ph. Bénilan. Equations d'évolution dans un espace de Banach quelconque et applications. Thesis, Univ. Orsay, 1972. 
[6] Ph. Bénilan and M. G. Crandall. Completely accretive operators. In Semigroup theory and evolution equations (Delft, 1989), volume 135 of Lecture Notes in Pure and Appl. Math., pages 41-75. Dekker, New York, 1991.

[7] Ph. Bénilan, M. G. Crandall and A. Pazy. Evolution Equations Governed by Accretive Operators. Book to appear.

[8] H. Brezis. Problèmes Unilatéraux. J. Math. Pures et Appl., 51, 1-169, (1992).

[9] C. Carrillo and P. Fife. Spatial effects in discrete generation population models. J. Math. Biol. 50(2), 161-188, (2005).

[10] E. Chasseigne, M. Chaves and J. D. Rossi. Asymptotic behaviour for nonlocal diffusion equations. To appear in J. Math. Pures Appl.

[11] X Chen. Existence, uniqueness and asymptotic stability of travelling waves in nonlocal evolution equations. Adv. Differential Equations, 2, 125-160, (1997).

[12] C. Cortazar, M. Elgueta, J.D. Rossi and N. Wolanski. Boundary fluxes for non-local diffusion. Preprint.

[13] C. Cortazar, M. Elgueta, J.D. Rossi and N. Wolanski. How to approximate the heat equation with Neumann boundary conditions by nonlocal diffusion problems. Preprint.

[14] M. G. Crandall. An introduction to evolution governed by accretive operators. In Dynamical systems (Proc. Internat. Sympos., Brown Univ., Providence, R.I., 1974), Vol. I, pages 131165. Academic Press, New York, 1976.

[15] C. M. Dafermos. Asymptotic Behavior of Solutions of Evolution Equations. In Nonlinear Evolution Equations (M. G. Crandall , eds.) Academic Press, 1978, pp.103-123.

[16] P. Fife. Some nonclassical trends in parabolic and parabolic-like evolutions. Trends in nonlinear analysis, 153-191, Springer, Berlin, 2003.

[17] P. Fife and X. Wang. A convolution model for interfacial motion: the generation and propagation of internal layers in higher space dimensions. Adv. Differential Equations 3(1), 85-110, (1998).

[18] L. Silvestre. Hölder estimates for solutions of integro differential equations like the fractional laplace. Indiana Univ. Math. J. 55(3), 1155-1174, (2006).

[19] X. Wang. Metaestability and stability of patterns in a convolution model for phase transitions. J. Differential Equations, 183, 434-461, (2002).

[20] L. Zhang. Existence, uniqueness and exponential stability of traveling wave solutions of some integral differential equations arising from neuronal networks. J. Differential Equations 197(1), 162-196, (2004).

Fuensanta Andreu, Jose M. Mazón and J. Julián Toledo-Melero Departament de Matemàtiques, Universitat de València

VALENCIA, SPAIN.

E-mail address: fuensanta.andreu@uv.es, mazon@uv.es, toledojj@uv.es

Julio D. Rossi

Instituto de Matemáticas y Física Fundamental

Consejo Superior de Investigaciones Científicas

SERrano 123, MADrid, Spain,

on leave from Departamento de Matemática, FCEyn UBA (1428)

Buenos Aires, Argentina.

E-mail address: jrossi@dm.uba.ar 